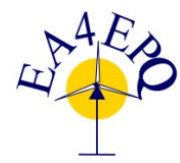

International Conference on Renewable Energies and Power Quality (ICREPQ'13)

Bilbao (Spain), 20 ${ }^{\text {th }}$ to $22^{\text {th }}$ March, 2013

Renewable Energy and Pourer Qualiny. Fournal (RE\&PQJ)

ISSN 2172-038 X, No.11, March 2013

\title{
Modelling and Simulation of Hierarchical Control for AC Inductive Microgrids
}

\author{
A. Martin-Villate ${ }^{1}$, J. Vadillo ${ }^{1}$, J.P. Fossati ${ }^{1}$, L. Arrizubieta ${ }^{2}$ and I. Cerro ${ }^{2}$ \\ ${ }^{1}$ Electronics and Communications Department \\ CEIT and Tecnun (University of Navarra) \\ Paseo de Manuel Lardizábal 15, 20018 San Sebastián, Spain \\ Phone: +0034 9432128 00, e-mail: amvillate@ceit.es, jvadillo@ceit.es, jpfossati@ceit.es \\ 2 JEMA \\ Paseo del Circuito 10, 20160 Lasarte-Oria, Spain \\ Phone: +0034943 3764 00, e-mail: l.arrizubieta@jema.es, i.cerro@jema.es
}

\begin{abstract}
This paper deals with the design of a hierarchical control system for an AC microgrid (MG). This control has been specifically implemented and validated in simulations for i-Sare MG project [1]. During the last years, great efforts have been done trying to adapt the hierarchical control defined for large power systems to the MG control. In this context, this paper presents and analyzes a four level hierarchical control for MGs based on droop control. Due to inherent drawbacks of droop control, this paper mainly focuses on Secondary and Tertiary control in order to minimize its limitations. For that purpose, simulation models have been implemented based on MG literature but modified and optimized for i-Sare requirements and specifications. These models allow to analyze the MG performance under any set point change. In order to show the reliability of the models, a simplified MG system which is composed of two inverters connected in parallel to the load is simulated and analyzed.
\end{abstract}

\section{Key words}

Microgrid, distributed generation, hierarchical control and droop control.

\section{Introduction}

Nowadays, electrical grids tend to be more distributed, intelligent and flexible. They are not only driven by the growing environmental concern and the energy security, but also by the liberalization of the electricity market. Instead of traditional producer-centralized power systems, the new electrical grid, also called smart grid (SG) tend to be more distributed, and consequently, energy generation and consumption areas cannot be conceived separately. Hence, microgrids [2] are becoming a reality to cope with a new scenario in which renewable energy, distributed generation (DG) and distributed energy-storage systems have to be integrated together. This new concept makes the final user not to be a passive element in the grid, but an entity able to generate, storage, control and manage part of the energy that he/she will consume. Besides, a reduction in cost and an increment in reliability and transparency are achieved.

The observed radical transformation of the electrical grid entails deep challenges not only on the architecture of the power system, but also in the control system. There are many control techniques in the literature based on the droop control method whose aim is to avoid communication between DG units [3] - [8]. Although this method achieves good reliability and flexibility, it presents several drawbacks [9], [10]: 1) it is not suitable when parallel DG units share nonlinear loads, it must take into account harmonic currents; 2) the output impedance of DG units and the line impedances affect the power sharing accuracy; 3 ) it is dependent of the load frequency deviations, which implies a phase deviation between DG units and grid/load. To cope with this problem, two additional control loops have been defined in [11] and [12]: Secondary control, which restores the nominal values of the frequency and voltage in the MG; and Tertiary control, which sets the reference of the frequency and voltage in the MG.

The aim of this paper is to test in simulations the Secondary and Tertiary control loop of a simplified MG which will be part of the i-Sare project mentioned before. This MG will be used as a platform in which new products, equipment, systems and procedures for operation and maintenance will be tested by the company participants in the i-Sare project. The power generation capability of the MG to be implemented will be about $500 \mathrm{~kW}$. A set of loads, generators and storage systems, which will constitute the MG, could operate connected to the main grid or in island mode.

This paper is organized as follows. In Section 2, the hierarchical control [13] of i-Sare AC MG is described. In Section 3, the Secondary and Tertiary control models are presented in Simulink. In Section 4, simulation results 
are presented, and finally, in Section 5, conclusions are drawn and future trends are defined.

\section{Hierarchical Control of i-Sare AC MG}

In order to implement the simplified MG, two essential requirements have to be fulfilled. On one hand, the $\mathrm{MG}$ should combine both different production technologies of renewable energy and conventional generation sources, as well as intelligent storage systems. And on the other hand, a smart control system of the MG has to be developed to control the performance of the whole system.

Renewable energy sources to be considered in the project will be: wind micro-turbine with horizontal axis of two blades, wind micro-turbine with horizontal axis of three blades, organic photovoltaic panels, conventional photovoltaic panels and fuel cell. From the conventional sources, the generation elements will be: gas micro-turbine and diesel group. Finally, lithium ion batteries sealed lead batteries, supercapacitors and flywheels will be considered for energy storage.

In this framework, in order to manage the MG composed of all these systems we propose a four-level control system which fulfills the requirements described above:

1) Level 1: Primary Control: It is the responsible of the droop-control method, which is used in this level to emulate physical behaviours. The aim is to maintain the system stable and more damped. It can include a virtual impedance control loop to emulate physical output impedance.

2) Level 2: Secondary Control: This control is able to modify the deviations in frequency and amplitude originated in the MG in order to keep it stable and capable of coping with demand. In addition, it can include a synchronization control loop to connect or disconnect the MG to or from the distribution system, an unbalance compensation control loop or a harmonics suppression control loop to enhance the power quality, which can be used in both islanded and grid-connected operation mode.

3) Level 3: Tertiary Control: This level sets the references of frequency and amplitude for each node of the MG in order to satisfy with $\mathrm{P}$ and $\mathrm{Q}$ requirements imposed by quaternary control.

4) Level 4: Quaternary Control: This level is responsible for optimal management of the energy resources available in the MG. In order to obtain an efficient use of the available renewable energy sources and storage systems, from the active $(P)$ and reactive $(Q)$ power demanded by the $\mathrm{MG}$, this control generates $P$ and $Q$ references to be required to each node of the system. A node is defined as any generation, storage or loading system in the MG.

This paper is focused on how to implement and use the Secondary and Tertiary control, based on Droop Control Method [14], to obtain a stable MG operating in island mode.

\section{Secondary and Tertiary control loop description}

The aim of this section is to implement a flexible and stable control based on droop method, which can manage the resources available in the MG in a stable and efficient way.

\section{A. Active Power Control}

In large scale power systems, AC generators drop their frequency when the output power increases due to their own inertia. This inertia is desirable because it makes the MG more stable. Nevertheless in small systems like batteries or supercapacitor, among others, it is negligible. So we use the droop method to provide those systems the desirable inertia:

$$
w=w_{\text {tertiary }}-\frac{\Delta w}{P_{\max }} \cdot P_{\text {measure }}+\delta w
$$

Where $w_{\text {tertiary }}$ is the reference given by the Tertiary control; $\Delta w$ is the maximum frequency deviation allowed (see Fig. 1); $\mathrm{P}_{\max }$ is the maximum active power that can be delivered by the inverter; $P_{\text {measure }}$ is the active power given by the inverter; and $\delta w$ is the compensation for frequency deviations given by the Secondary control.

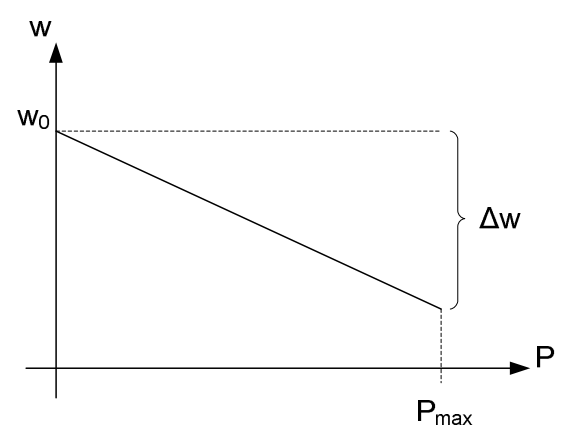

Fig. 1. Static droop characteristics P-w.

In order to obtain the $w_{\text {tertiary }}$ and $\delta w$, the Tertiary and Secondary control are used respectively.

As mentioned above, the Tertiary control sets the frequency reference for each MG node in order to satisfy the requirement of $P$. It is assumed that the output impedance of the generators is mainly inductive. Hence, the droop control establishes a direct relationship between $P$ and $w$. The control law $P I_{P}$ shown in Fig. 2, can be expressed as:

$$
w_{n}=k_{p P_{-} n}\left(P_{n}^{*}-P_{n}\right)+k_{i P_{-} n} \int\left(P_{n}^{*}-P_{n}\right) d t
$$

Where the subscript $n$ refers to the number of nodes in the MG; $P_{n}^{*}$ is the desired reference value given by the quaternary control; $P_{n}$ is the measured value; and $k_{p P \_n}$ and $k_{i P \_n}$ are the control parameters of the Tertiary control compensator. 
On the other hand, the control law of Secondary control, described in Fig. 2, is able to modify the frequency deviations originated in the MG. It can be expressed as:

$$
\delta w_{n}=k_{p w_{-} n}\left(w_{n}-w_{n}^{*}\right)+k_{i w_{-} n} \int\left(w_{n}-w_{n}^{*}\right) d t+\Delta w_{s}
$$

Where the subscript $n$ refers to the number of nodes in the MG; $w_{n}$ is the desired frequency reference value given by the Tertiary control; $w_{n}{ }_{n}$ is the desired frequency in the MG, (in grid-connected operation it is given by the main grid, and in islanded operation it is set arbitrarily); and $k_{p w \_n}$ and $k_{i w_{-} n}$ are the control parameters of the Secondary control compensator.

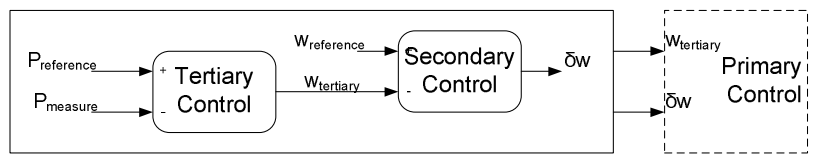

Fig. 2. Active Power Control

\section{B. Reactive Power Control}

As mentioned above, the inertia that large scale systems present, is also desirable in small systems like batteries or supercapacitors, because it makes the MG more stable. So we use the droop control method to provide those systems the desirable inertia:

$$
E=E_{\text {tertiary }}-\frac{\Delta E}{Q_{\max }} \cdot Q_{\text {measure }}+\delta E
$$

Where $E_{\text {tertiary }}$ is the reference giving by the Tertiary control; $\Delta E$ is the maximum allowed voltage amplitude deviation (see Fig. 3); Qmax is the maximum reactive power that can be delivered by the inverter; $Q_{\text {measure }}$ is the reactive power given by the inverter; and $\delta E$ is the compensation for voltage amplitude deviations given by the Secondary control.

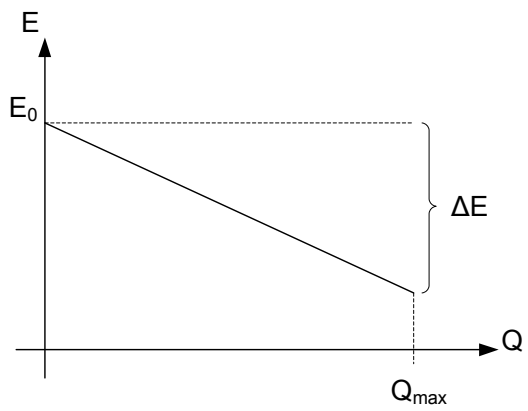

Fig. 3. Static droop characteristic Q-E.

To obtain the $E_{\text {tertiary }}$ and $\delta E$ it is used the Tertiary and Secondary control respectively, as it is described as follows.

Firstly, the Tertiary control sets the amplitude reference of each MG node in order to satisfy the requirements of $Q$. The control law (see Fig. 4) can be expressed by:

$$
E_{n}^{*}=k_{p Q_{-} n}\left(Q_{n}^{*}-Q_{n}\right)+k_{i Q_{-} n} \int\left(Q_{n}^{*}-Q_{n}\right) d t
$$

Where the subscript $n$ refers to the number of nodes of the MG; $Q^{*}{ }_{n}$ is the desired reference value given by the Quaternary control; $Q_{n}$ is the measured value; and $k_{p Q \_n}$ and $k_{i Q \_n}$ are the control parameters of the Tertiary control compensator.

Secondly, the Secondary control modifies the deviations in voltage amplitude originated in the $\mathrm{MG}$ in order to keep the MG stable. The control law (see Fig. 4) can be expressed by:

$$
\delta E_{n}=k_{p E_{-} n}\left(E_{n}^{*}-E_{n}\right)+k_{i E_{-} n} \int\left(E_{n}^{*}-E_{n}\right) d t
$$

Where the subscript $n$ refers to the number of nodes of the MG; $E_{n}^{*}$ is the desired reference value, given by the Tertiary control; $E_{n}$ is the measured voltage; and $k_{p E_{\_} n}$ and $k_{i E \_n}$ are the control parameters of the Secondary control compensator.

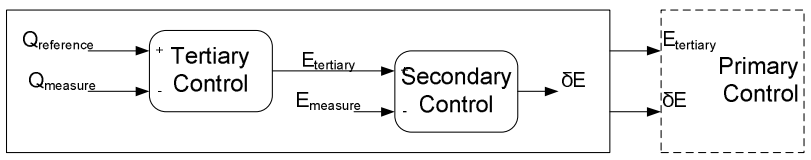

Fig. 4. Reactive Power Control

\section{Simulation Results}

The proposed control implementation was simulated in a simplified model composed of two inverters working in parallel sharing a load, so that the feasibility of the proposed hierarchical control could be observed. The MG rms voltage was selected at $220 \mathrm{~V}$.

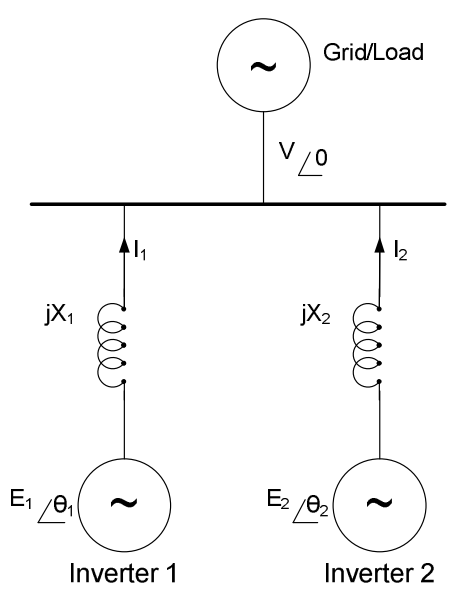

Fig. 5. Equivalent circuit of two MG nodes supplying a common load.

The voltage waveform which will be the reference of the PWM inverter, is the waveform to be consider in the MG control analysis:

$$
E_{\text {inverter }}=E^{*} \sin e\left(w^{*} t\right)
$$

Where $E$ is obtained from equation (1) and $w$ from equation (4), according to proposed Tertiary and Secondary control. 

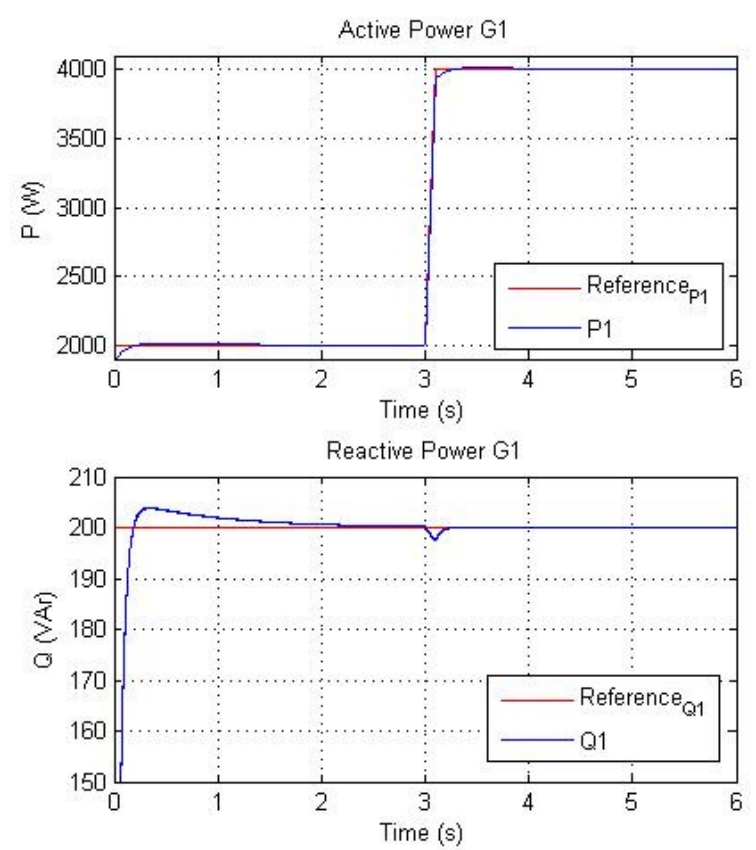
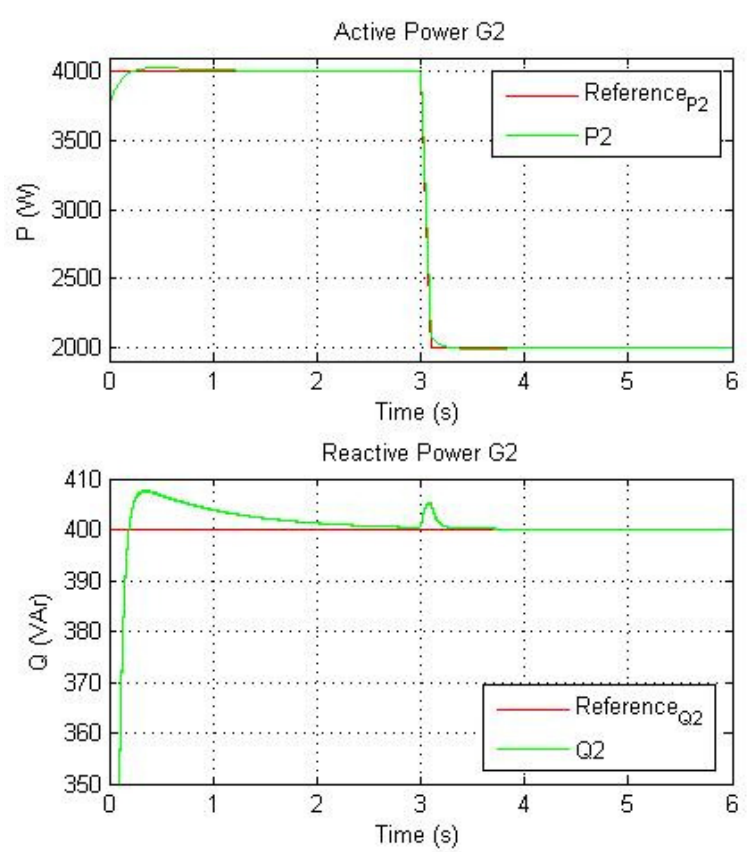

Fig. 6. Active and reactive power waveform of inverter 1 and 2.

\section{A. Changing Active Power}

Fig. 6 depicts the transient response of a two parallel inverter system at the start up, and also when the active power reference of the inverter change at $t=3 \mathrm{~s}$. It can be seen a good transient response with a small overshoot in both active and reactive power for both inverters of the MG.

Fig. 7 and Fig. 8 show how the Secondary control loop compensates the generated frequency and amplitude deviation when active power reference of inverters is changed.

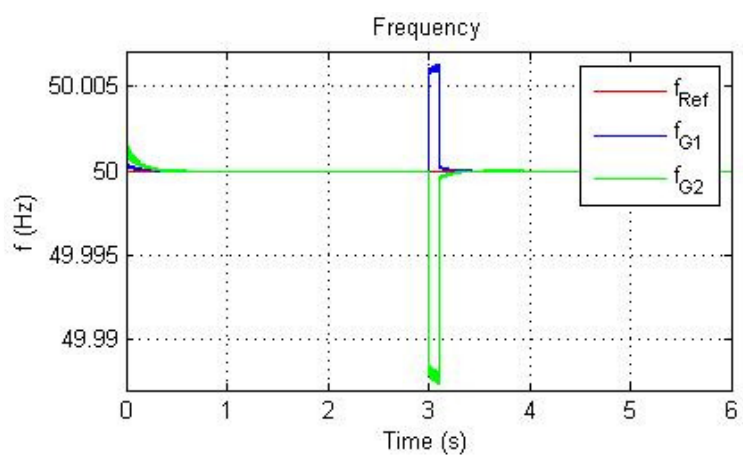

Fig. 7. Frequency transient response.

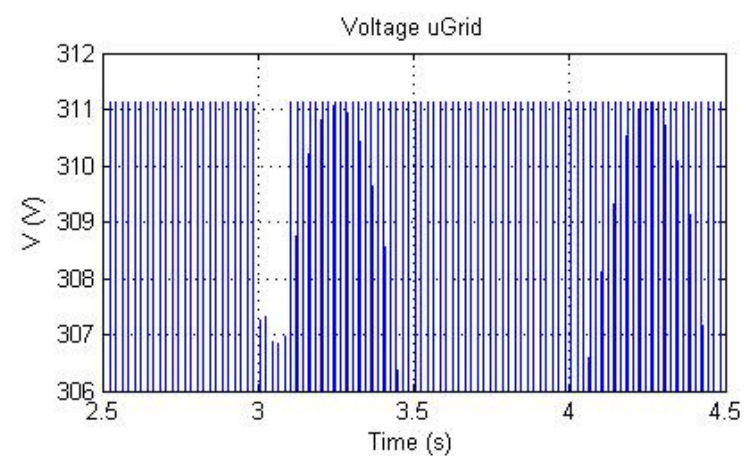

Fig. 8. Voltage transient response of the ac MG.
This frequency deviation causes small changes in the phase difference between the inverter output and the MG, consequence of the power delivery or absorption in the MG. At the same time a small change in the output voltage amplitude of the inverters is also provoked, as it can be seen in Fig. 9 .

Although the theory of droop control method considers that it does not exist coupling between active and reactive power, this is not completely true. In the simulated system, it can be seen a small coupling between the active and reactive power that can be ignored if the output impedance of the inverter is purely inductive. As is shown in Fig. 9, the voltage amplitude change in the inverter is very small. It can also be observed in Fig. 8 that there is not MG amplitude voltage deviation after frequency stabilization.

Despite the fact that the active power load has not been changed (power references have been modified in order to simulate a time varying load change), the presented model and derived figures confirm that the proposed controller is stable, flexible and reliable, showing a fast response after any change of active power reference.

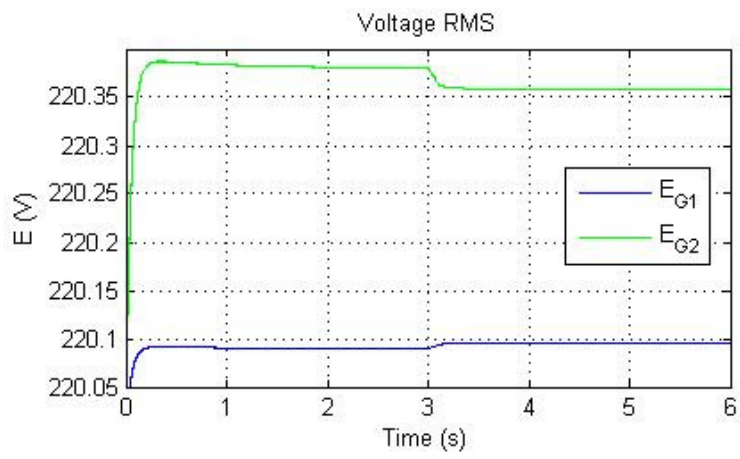

Fig. 9. Voltage transient response in the inverters. 


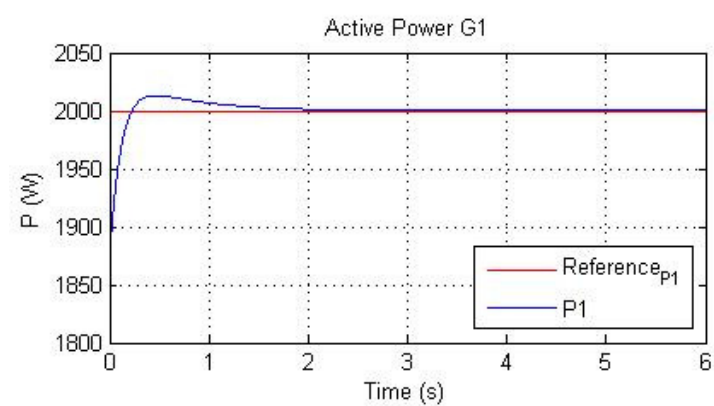

Reactive Power G1

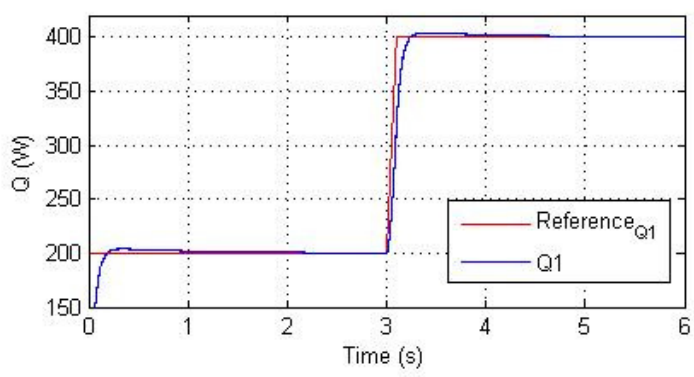

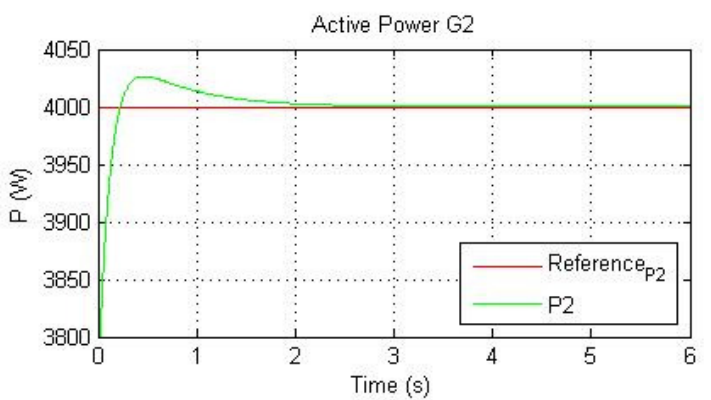

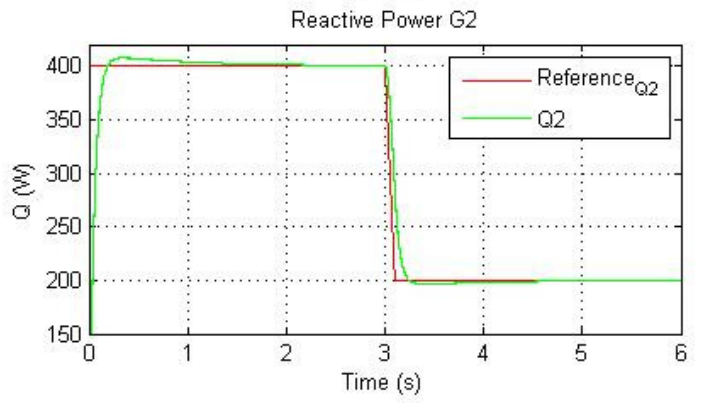

Fig. 10. Active and reactive power waveform of inverter 1 and 2 .

\section{B. Changing Reactive Power}

Firstly, Fig. 10 depicts the transient response of a two parallel inverter system at the startup, and also when the reactive power reference of the inverter change at $t=3 \mathrm{~s}$. As in the previous case, it can be seen a good transient response with a small overshoot in both active and reactive power for both inverters of the MG.

How the Secondary control loop compensates generated frequency deviation when reactive power reference of inverters is changed, is shown in Fig. 11.

Fig. 12 shows how there is no change in the MG voltage amplitude during power adjustments. This is because there has been no change in the load, there has only been a change in the distribution of reactive power. If there had been a change in the load, a little change of the MG voltage amplitude would have been observed.

Finally, Fig. 13 show how the voltage Tertiary control sets new voltage reference for inverters when a new reactive power value is fixed, as can be seen in Fig. 10.

As previously mentioned in the active power analysis, load changes have been modelled balancing power references, obtaining stable and fast transient operation.

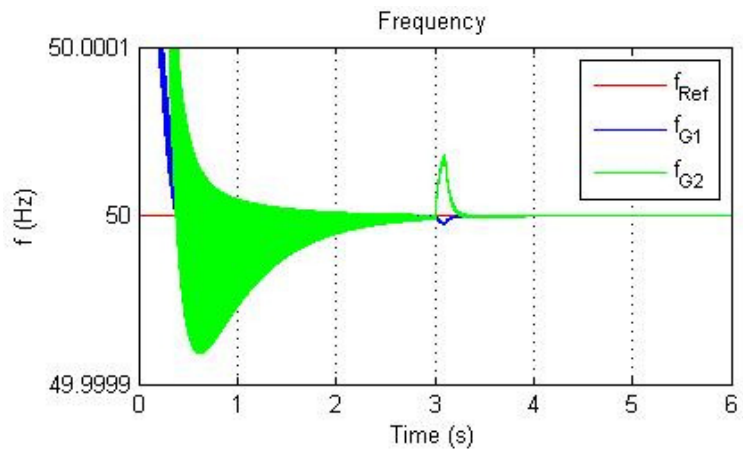

Fig. 11. Frequency transient response.

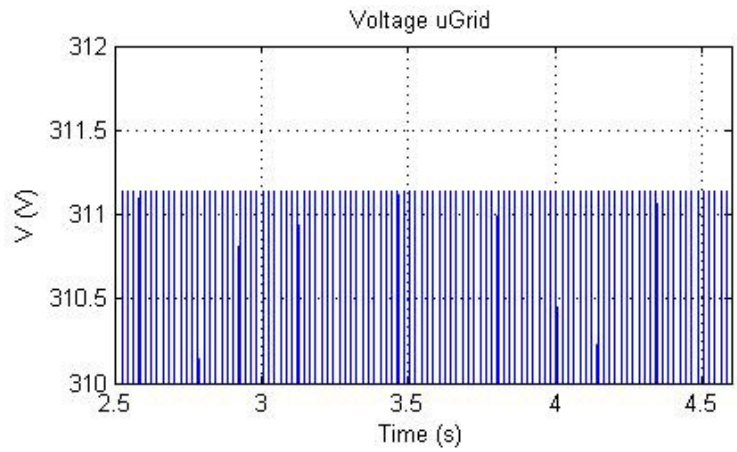

Fig. 12. Voltage transient response of the ac MG.

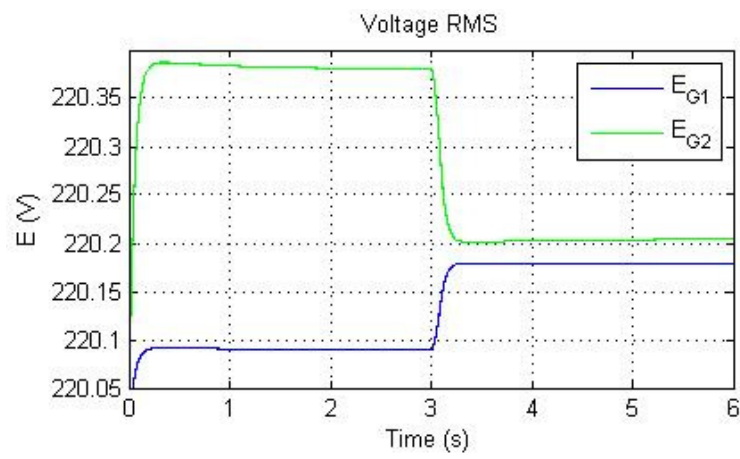

Fig. 13. Voltage transient response of inverters.

\section{Conclusion}

Results from presented simulations validate the feasibility of the proposed Secondary and Tertiary control cascade implementation based on droop control approach. This way, these two additional control loops allow getting over the droop control method limitations. Results from simulations show that Secondary and Tertiary control provide the necessary stability to the MG, making it flexible and reliable when several DG units must be connected to a common AC bus. Furthermore, it can be observed that a correct tuning of 
the control makes the transient response accurate and fast.

Taking into consideration the proposed models and simulation results presented in this paper, an experimental validation is intended to be achieved by means of a small MG composed of two paralleled inverters connected to the load.

\section{Acknowledgement}

The authors would like to thank JEMA for offering the possibility of this research work, their technical collaboration and financial support. They also wish to express their gratitude to Gipuzkoa Aurrera, Gipuzkoa Provincial Government (Diputación Foral de Gipuzkoa), the Ministry of Economy and Competitiveness, GAIACluster TEIC and IK4 for their financial support of i-Sare project.

\section{References}

[1] http://www.i-sare.net/pages/quees.html

[2] R. H. Lasseter et al., "White paper on integration of distributed energy resources. The CERTS microgrid concept," in Proc. Consortium Elect. Reliab. Technol. Solutions, 2002, pp. 1-27.

[3] Barsali, S.; Ceraolo, M.; Pelacchi, P.; Poli, D.; , "Control techniques of Dispersed Generators to improve the continuity of electricity supply," Power Engineering Society Winter Meeting, 2002. IEEE, vol.2, no., pp. 789- 794 vol.2, 2002

[4] Sung-Woo Park; Il-Yop Chung; Joon-Ho Choi; Seung-Il Moon; Jae-Eon Kim; , "Control schemes of the inverter-interfaced multi-functional dispersed generation," Power Engineering Society General Meeting, 2003, IEEE, vol.3, no., pp. 1924- 1929 Vol. 3, 13-17 July 2003

[5] R. H. Lasseter, "Microgrids," in Proc. IEEE PES'02 Winter Meeting, 2002, pp. 305-308.

[6] C.-C. Hua, K.-A. Liao, and J.-R. Lin, "Parallel operation of inverters for distributed photovoltaic power supply system," in Proc. IEEE PESC'02 Conf., 2002, pp. 1979-1983.

[7] J. Sachau and A. Engler, "Static and rotating grid formation for modularly expandable island grids," in Proc. EPE'99 Conf., Laussane, Frane, 1999, pp. 110.

[8] H. Matthias and S. Helmut, "Control of a three phase inverter feeding an unbalanced load and operating in parallel with other power sources," in Proc. EPEPEMC'02 Conf., 2002, pp. 1-10.

[9] A. Tuladhar, H. Jin, T. Unger, and K. Mauch, "Parallel operation of single phase inverter modules with no control interconnections," in Proc. IEEE APEC'97, 1997, pp. 94-100.

[10] Tuladhar, A.; Hua Jin; Unger, T.; Mauch, K.; "Control of parallel inverters in distributed AC power systems with consideration of line impedance effect," Industry Applications, IEEE Transactions on, vol.36, no.1, pp.131-138, Jan/Feb 2000

[11] Guerrero, J.M.; Vasquez, J.C.; Matas, J.; Castilla, M.; de Vicuna, L.G.; , "Control Strategy for Flexible Microgrid Based on Parallel Line-Interactive UPS
Systems," Industrial Electronics, IEEE Transactions on, vol.56, no.3, pp.726-736, March 2009

[12] Guerrero, J.M.; Vasquez, J.C.; Matas, J.; de Vicuna, L.G.; Castilla, M.; , "Hierarchical Control of DroopControlled AC and DC Microgrids-A General Approach Toward Standardization," Industrial Electronics, IEEE Transactions on, vol.58, no.1, pp.158-172, Jan. 2011

[13] Vasquez, J.C.; Guerrero, J.M.; Miret, J.; Castilla, M.; de Vicuña, L.G.; , "Hierarchical Control of Intelligent Microgrids" Industrial Electronics Magazine, IEEE, vol.4, no.4, pp.23-29, Dec. 2010

[14] Guerrero, J.M.; de Vicuna, L.G.; Matas, J.; Castilla, M.; Miret, J.; , "A wireless controller to enhance dynamic performance of parallel inverters in distributed generation systems," Power Electronics, IEEE Transactions on, vol.19, no.5, pp. 1205- 1213, Sept. 2004 